\title{
Pericardial Tamponade as the First Manifestation of Breast Cancer
}

Sara Belabyad*, Ali Laalou, Joumana El Masrioui, Dounia Benzeroual, Saloua El Karimi, Mustapha El Hattaoui

Cardiology Department, Mohammed VI University Hospital, Marrakesh

DOI: $10.36347 /$ sjmcr.2020.v08i03.029

| Received: 10.02.2020 | Accepted: 18.02.2020 | Published: 19.03.2020

*Corresponding author: Sara Belabyad

\section{Abstract}

Neoplastic involvement of the pericardium occurs frequently in post-mortem studies. Symptoms of this involvement of the pericardium prior to death are unusual. This report describes a case of breast carcinoma in a 41 -year-old woman who presented with cardiac tamponade. Because breast carcinoma as a cause of cardiac tamponade is unusual, its presentation is reported. Early recognition of the possibility of malignancy allows for initiation of appropriate treatment, thereby improving the patient's chance of survival. It is recommended that all patients who present with tamponade have pericardial fluid sent for cytologic examination.

Keywords: Pericardial effusion, cardiac tamponade, breast cancer.

Copyright @ 2020: This is an open-access article distributed under the terms of the Creative Commons Attribution license which permits unrestricted use, distribution, and reproduction in any medium for non-commercial use (NonCommercial, or CC-BY-NC) provided the original author and source are credited.

\section{INTRODUCTION}

Breast cancer rarely metastasizes to the pericardial cavity to cause cardiac Tamponade [1]. Although malignant pericardial and pleural effusions are common complications during the course of malignancies, they are rarely the initial manifestations of malignant disease, particularly pericardial effusion with cardiac tamponade. Pleural effusion is the first symptom of metastatic disease. The prevalence of pericardial involvement varies from $4 \%$ in general autopsies to $15-30 \%$ in autopsies of cancer patients. Only $12-25 \%$ of patients who have metastasis to the pericardium develop pericardial effusion of whom only a small percentage develop tamponade. Therefore, there are few reported cases of cardiac metastases from breast cancer. Pericardial effusion with cardiac tamponade as the initial manifestation of breast cancer is quite rare [1].

We describe a unique case of a young female with undiagnosed breast cancer presenting with cardiac tamponade.

\section{Case Report}

A 41 year old woman presented to the emergency complaining of progressive dyspnea, chest pain and dry cough accompanied by general malaise for 1 month, complicated by orthopnea 24 hours before admission .There was no associated nausea or vomiting, but she did experience some sweating with activity. The patient's past medical history was not significant.
On admission the patient was afebrile and in a moderate degree of respiratory distress. Her pulse was 110 beats per minute and regular, and her blood pressure was $105 / 82 \mathrm{mmHg}$. A paradoxical pulse of 20 $\mathrm{mmHg}$ was noted. The heart sounds were distant and faint. Jugular venous distention was observed. Chest auscultation was normal.

An electrocardiogram (ECG) revealed low voltage (Fig 1). Transthoracic echocardiography demonstrated a circumferential pericardial effusion of great abundance of $35 \mathrm{~mm}$ in anterior and $20 \mathrm{~mm}$ in posterior, with a swinging heart aspect and significant respiratory variations. Based on these findings a diagnosis of pericardial effusion with cardiac tamponade was made. An urgent pericardiocentesis was performed bringing approximately $800 \mathrm{ml}$ of serohematic fluid. After pericardiocentesis, the patient made a dramatic clinical improvement. Additionally, there was no longer a paradoxical pulse. An increase in electrical voltage on ECG was evident. The cytological examination of the pericardial effusions demonstrated a sterile liquid without atypical cells.

After stabilization, an etiological assessment has been carried out. Mammography showed diffuse infiltration of the right breast with suspicious bilateral axillary lymphadenopathy. 
on the thoraco abdomino pelvic CT scan we noted an aspect of right carcinomatous mastitis associated with homolateral axillary lymphadenopathy and thrombosis of the superior vena cava extended to innominate venous trunks, internal jugular veins, external jugular vein and right subclavian, with Mediastinal lymphadenopathy flows associated with pleuropericarditis, pulmonary nodules and micronodules, secondary liver and bone damage. At this time, tamponade was thought to be most probably due to metastatic breast carcinoma.
A pleuropericardial window was performed after stabilization, and the pericardium and epicardial surface of the heart were seen to be grossly involved by metastatic lesions. The anatomopathological examination of pericardial and pleural biopsies showed a slightly differentiated carcinoma infiltrating the pleural and pericardial parenchyma with vascular emboli and whose appearance can fit into a metastasis from a mammary primitive.

The patient was treated with low molecular weight heparin 0,6 UIx2/D. Chemotherapy indicated but the evolution was marked by death.

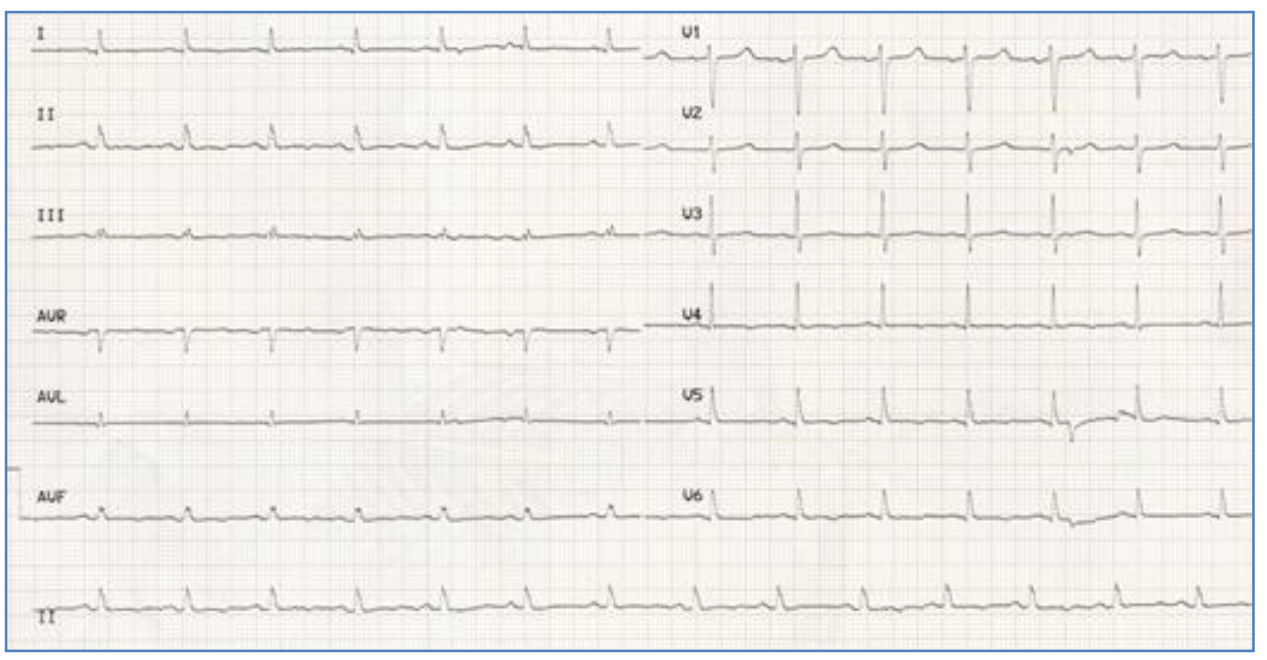

Fig-1: Electrocardiogram showed a diffuse Microvoltage

\section{DiscuSSION}

Cardiac metastasis occurs frequently in neoplastic disease. It has been shown that from 1 to 18 percent of post mortem studies have demonstrated metastasis. Still, it is rare to have this kind of cardiac impairment during a patient's lifetime. There are three routes that result in extension of malignancy to the pericardium: hematogenous, lymphatic, and local extension [2]. The most common malignancy associated with metastasis to the pericardium is the lung $[2,3]$.

Breast cancer is the most prevalent malignancy in the female population. Metastatic disease of the pericardium resulting from the breast in unusual. This appears to be because breast cancer usually spreads to regional axillary lymph nodes before disseminating to other locations. Lymphatic and local extension are two mechanisms that are less common in breast cancer. Therefore, breast carcinoma is not a frequent cause of cardiac metastasis [3].

Neoplastic obstruction of venous and lymphatic drainage of the heart disrupts the equilibrium existing between capillary filtration, hydraulic, and osmotic forces, resulting in excessive accumulation of pericardial fluid. In addition, metastatic implants on pericardial serosal surfaces cause fluid exudation. The consequences of pericardial fluid accumulation are largely dependent on the rate of exudation, the compliance of the pericardial cavity, the myocardial mass, and the blood volume of the patient. Extremely large effusions are well tolerated if they accumulate slowly in a patient with a compliant pericardium, a thick myocardium, and a high blood volume. However, rapid accumulation of fluid inside a thickened pericardium rendered stiff by tumor infiltration or radiation fibrosis generally progresses to cardiac Tamponade [4]. The hemodynamic consequences of pericardial fluid accumulation result from impaired diastolic filling of the heart, leading to decreased cardiac output. Symptoms may arise gradually or rapidly, depending on fluid accumulation rate, and may range from dyspnea, chest pain, cough, palpitations and orthopnea, to fatigue, anxiety and confusion. At physical examination, tachycardia, decreased heart sounds, neck vein distension, peripheral oedema and pericardial friction rubs may be present. Pulsus paradoxus, defined as an inspiratory decrease of more than $10 \mathrm{mmHg}$ in systolic pressure, is a rare but suggestive sign of pericardial effusion. As cardiac tamponade develops, patients may show signs of lowoutput shock, cardiac arrest, and death rapidly ensue [4]. 
Metastatic tumors to the heart resulting in malignant pericardial effusion with cardiac tamponade as the initial clinical sign are not seen frequently. Our patient showed cardiac tamponade as the initial clinical sign of breast carcinoma.

At initial evaluation, a chest radiograph may provide evidence of an enlarged cardiac silhouette, while at electrocardiography (ECG) low-voltage complexes and electrical alternans are suggestive of pericardial effusion. However, two-dimensional echocardiography is considered the standard method for diagnosing pericardial effusion.

Acute pericardial tamponade with life threatening hemodynamic instability must be alleviated by prompt withdrawal of fluid. This is most rapidly accomplished by pericardiocentesis. Clearly, pericardiocentesis is the first step in treating cardiac tamponade, as a reduction in the malignant effusion will often relieve symptoms quickly. Aggressive intravenous fluid support and administration of a sympathomimetic agent (particularly isoproterenol hydrochloride) can be employed as temporizing measures to support cardiac output until transfer of the patient to a controlled environment is possible [4]. Fluid aspiration ideally should be done in association with fluoroscopy and right-sided heart catheterization. In virtually all cases, pericardiocentesis should be preceded by echocardiographic confirmation of pericardial effusion, and electrocardiographic guidance of the exploring needle should be employed to prevent cardiac puncture $[4,5]$. Hazards associated with the procedure are markedly enhanced in patients with thrombocytopenia, small effusions $(<200 \mathrm{~mL})$, loculated effusions, or presumptive effusions unconfirmed by echocardiography $[6,7]$. The risks of pericardiocentesis have prompted some authors to abandon the procedure in favor of subxiphoid pericardiotomy, an operation that can be performed in 45 minutes under local anesthesia with negligible morbidity or mortality $[8,9]$.

Simple pericardiocentesis alone is effective in the emergent management of neoplastic tamponade, but rapid reaccumulation of fluid generally occurs unless more definitive measures are subsequently employed [4], treatment alternatives include radiotherapy [11, 12], intrapericardial sclerosing agents [11], intrapericardial chromic phosphate [13], pleuropericardial window [14], and finally, systemic therapy alone [15]. The treatment of choice remains to be identified, and the use of systemic chemotherapy can be debated. Obviously, the treatment decision must be based on the tumor type and the long-term prognosis of the individual patient.

The preponderance of the reviewed data suggests that acute neoplastic cardiac tamponade can be effectively relieved by pericardiocentesis in approximately $90 \%$ of cases [4, 7], with acceptable risk (approximately $1 \%$ major complications) if the procedure is performed in a controlled environment with fluoroscopic and electrocardiographic guidance and follows confirmation of pericardial fluid by echocardiography. Subxiphoid pericardiotomy is an excellent, $100 \%$ effective, safe alternative that can be performed under local anesthesia in institutions experiencing an unacceptable complication rate with pericardiocentesis [4]. Long-term management of malignant pericardial effusions must be individualized.

The prognosis of cardiac tamponade caused by malignant pericarditis is grave, and survival is limited. Although no standard treatments for malignant pericardial effusion have yet been established. Pericardial effusion is often resistant to treatments; thus, it results in poor prognosis. Furthermore, it requires a repetitive administration of sclerosing reagents, and treatment related complications are known $[11,15]$.

The treatment of cardiac tamponade in patients with disseminated breast cancer presents special considerations. First, many active single chemotherapeutic drugs and combinations have been identified. Second, survival in patients with stage IV breast carcinoma is measured in years [15].

\section{CONCLUSION}

Cardiac tamponade may be the first clinical sign of primary breast cancer. This is a rare condition and difficult to diagnosis early. The prognosis is typically poor. Clinicians should be aware of these rare metastatic patterns of breast cancer.

\section{REFERENCES}

1. Xingxing lv, Jinlan He. malignant pleural and pericardial effusions and meningeal infiltrates without other metastases in breast cancer: A case report. Molecular and clinical oncology.2016; 4: $855-858$

2. Kline IK. Cardiac lymphatic involvement by metastatic tumor. Cancer. 1972; 29:799-808.

3. Van J. Stitt, Jr. Breast carcinoma with pericardial metastasis. Journal of the national medical association.1987; 79(2)

4. Oliver W. Press, Robert Livingston. Management of Malignant Pericardial Effusion and Tamponade. Jama, Feb 27. 1987;257(8)

5. Callaham ML. Pericardiocentesis in traumatic and non traumatic cardiac tamponade. Ann Emerg Med. 1984;13:924-945

6. Krikorian JG, Hancock EW: Pericardiocentesis. Is J Med. 1978; 65:808-814.

7. Wong B, Murphy J, Chang CJ and al: The risk of pericardiocentesis. Am J Cardiol. 1979; 44:11101114.

8. Reitknecht F, Regal AM, Antkowiak JG. Management of cardiac tamponade in patients with malignancy. J Surg Oncol. 1985; 30:19-22. 8. 
9. Alcan KE, Zabetakis PM, Marino ND. Management of acute cardiac tamponade by subxiphoid pericardiotomy. JAMA. 1982; 247:1143-1148.

10. Jacob d. Bitran, Richard Evans and al The Management of Cardiac Tamponade in Patients with Breast Cancer. Journal of Surgical Oncology.1984; 27:42-44

11. Mauch PM. Treatment of malignant pericardial effusions. In DeVita VT, Hellman S, Rosenberg SA (eds): "Cancer: Principles and Practice of Oncology." Philadelphia: JB Lippincott. 1982, 1571-1573
12. Chan WC, Freiman AH, Carstens PHB. Radiation therapy of cardiac and pericardial metastases. Radiology.1975; 114:701- 704

13. Martini N, Freeman AH, Walson RC. Intrapericardial instillation of radioactive chromic phosphate in malignant pericardial effusion. Am J Radio.1977; 128:639-645.

14. Hill GJ, Cohen BI. Pleural pericardial window for palliation of cardiac tamponade due to cancer. Cancer.1970; 26:81-93.

15. Reynolds PM, Byrne MJ. The treatment of malignant pericardial effusion in carcinoma of the breast. Aust NZ J Med.1977; 67:169-171. 